\title{
Gomputed Tomography in the Diagnosis of Fatty Liver: Total Lipid Content and Computed Tomography Number
}

\author{
Yoshiaki Yajima, Takashi Narui, Motoyasu IshiI, Ryuzo \\ Abe, Masao Ohtsuki, Yoshio Goto, Satoshi Endo, ${ }^{*}$ Kenji \\ Yamada* and Masatoshi Ito* \\ Third Department of Internal Medicine, Tohoku University \\ School of Medicine, and *Department of Radiology and Nuclear \\ Medicine, the Research Institute for Tuberculosis and Cancer, \\ Tohoku University, Sendai 980
}

Yajima, Y., Narui, T., Ishit, M., Abe, R., OHtsuki, M., Goto, Y., Endo, S., Yamada, K. and Iто, M. Computed Tomography in the Diagnosis of Fatty Liver: Total Lipid Content and Computed Tomography Number. Tohoku J. exp. Med., 1982, 136 (3), 337-342 — Fifty-three histologically proved cases of various diffuse liver diseases were studied for their computed tomography numbers (CTN). The machine used was Ohio Nuclear's Delta Scanner 50FS type and CTN was expressed by the Hounsfield unit $(\mathrm{H})$. Mean CTN in each group was as follows: $66.6 \pm 2.6 \mathrm{H}$ in normal control $(\mathrm{N}), 63.3 \pm 6.0 \mathrm{H}$ in chronic hepatitis $(\mathrm{CH}), 61.8 \pm 7.0 \mathrm{H}$ in liver cirrhosis $(\mathrm{LC})$, and $44.4 \pm 10.6 \mathrm{H}$ in fatty infiltration (FI). There were no significant differences among them except FI group. As N group were all above $60 \mathrm{H}$ and $\mathrm{CH}$ and $\mathrm{LC}$ groups were all above $50 \mathrm{H}$, CTN below $60 \mathrm{H}$ suggests chronic liver disease or fatty infiltration and CTN below $50 \mathrm{H}$ strongly suggests fatty infiltration. In eleven cases where total lipid content of the liver could be biochemically determined by the sulfo-phospho-vanillin reagent, a relation of total lipid content to CTN was studied. As a result, a significant correlation existed between them $(r=-0.89 ; p<0.001)$. If the diagnostic criterion of fatty liver was set at total lipid content above $100 \mathrm{mg} / \mathrm{g}$ wet liver, CT criterion was estimated at CTN below $48 \mathrm{H}$ from the regression formula. —— fatty liver; computed tomography; computed tomography number; total lipid content

The computed tomography has been studied in the diagnosis of diffuse liver diseases in order to apply it to the routine clinical practice. In this paper, the possibility of the diagnosis of fatty liver using computed tomography number (CTN) was discussed. The definition of fatty liver is ambiguous because histological criterion of fatty liver usually defines fatty infiltration above $50 \%$ of the hepatic lobule but some investigators define it above one-third of hepatic lobule (Okudaira 1980). Moreover, the estimation of the degree of fatty infiltration from the scattered fatty droplets in the hepatic tissue under the microscope seems to be rather a subjective process. Therefore, in this study, the term "fatty infiltration" was used preferably and cases of various degrees of fatty infiltration were

Received for publication, June 15, 1981. 
collected and total lipid content of the liver was measured biochemically. Then, the correlation between total lipid content and CTN in each case was investigated and CT criterion of fatty liver was estimated from the regression formula inversely.

\section{Methods}

Device. The machine used was Ohio Nuclear's Delta Scanner 50FS whole type. Either a 13-mm or an 8-mm thick slice can be obtained according to cases. The mean x-ray attenuation value $(\mathrm{CTN})$ in each pixel is expressed by Hounsfield unit $(\mathrm{H})$ : water in $0 \mathrm{H}$, bone about $+1000 \mathrm{H}$, and air $-1000 \mathrm{H}$. The scan was made at $130 \mathrm{kV}, 30 \mathrm{~mA}$ with a 3 $\mathrm{mm}$ aluminum filter.

Measurement of CTN. ROI (region of interest) program, one of the computer programs of the scanner, was used for calculating mean and standard deviation of the CTN in a given region of interest. Part of the liver immediately beneath the diaphragm was not suitable for CTN measurement because of its suffering from motion artifact of the heart. Therefore three slices in the center of the liver were taken in the measurement of mean CTNs and mode of them was adopted as CTN of a given case.

Determination of total lipid content of the liver. Biopsied specimens obtained by Silverman's needle were weighed immediately after biopsies and then frozen to be stored at $-20^{\circ} \mathrm{C}$. Later, lipid extracts were prepared after Folch's method (Folch et al. 1951) and total lipid content was determined by the sulfo-phospho-vanillin reaction (Frings et al. 1972).

Subjects. Patients with various diffuse liver diseases who were admitted to our Hospital and were subjected to CT Scan and liver biopsy coincidentally were chosen for the study, giving a total of 10 normal livers $(\mathrm{N}), 12$ with chronic hepatitis $(\mathrm{CH}), 18$ with liver cirrhosis (LC), and 13 with fatty infiltration (FI). In this study analysis of the effect of fatty infiltration on the CTN was emphasized, so the cases of chronic hepatitis or liver cirrhosis which had various degrees of fatty infiltration were included in the FI group.

Thirteen FI cases could be studied in the correlation between CTN and histological findings but only 9 cases could be analyzed about the total lipid content. Moreover, a case of $\mathrm{CH}$ and a case of LC, both of which showed no fatty infiltration were added to the study of total lipid content analysis and correlated to their CTNs.

Intervals between CT scanning and liver biopsy were within 2 weeks in all the cases. In the cases in which total lipid content could be measured, CT scanning was performed within a week, 4 days interval on average.

\section{RESULTS}

\section{CTN of various diffuse liver diseases}

Mean CTN in each group was as follows: $66.6 \pm 2.6 \mathrm{H}$ in $\mathrm{N}$ group, $63.3 \pm 6.0 \mathrm{H}$ in $\mathrm{CH}, 61.8 \pm 7.0 \mathrm{H}$ in $\mathrm{LC}, 44.4 \pm 10.6 \mathrm{H}$ in FI. There were no significant differences among them except FI group. As $\mathrm{N}$ group were all above $60 \mathrm{H}$ and $\mathrm{CH}$ and LC groups were all bove $50 \mathrm{H}$, CTN below $60 \mathrm{H}$ suggests chronic liver disease or fatty infiltration and CTN below $50 \mathrm{H}$ strongly suggests fatty infiltration (Fig. 1).

\section{Total lipid content of the various diffuse liver diseases}

The mean value of each group was as follows: $\mathrm{N}$ group $28.8 \pm 6.8, \mathrm{CH}$ group $33.8 \pm 8.9$, LC group $17.5 \pm 10.0$, FI group $83.1 \pm 45.9$ (mg/g wet liver). Total lipid content of $\mathrm{N}$ group was about $3 \%$ of the wet liver weight which conformed to 
other investigators (Kobayakawa 1959; Kalk 1965). FI group involved various degrees of cases from mild to severe fatty infiltration. so total lipid content showed a wide range of values from 38 to $190 \mathrm{mg} / \mathrm{g}$ wet liver. If the biochemical criterion of fatty liver was set at total lipid content above $10 \%$ of the wet liver weight, 4 cases among 13 FI cases were diagnosed as fatty liver (Fig. 2).

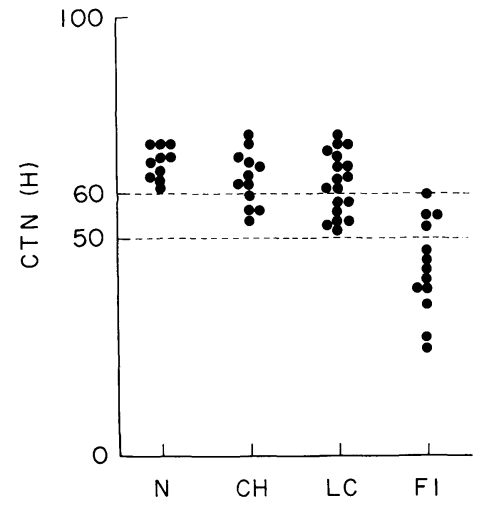

Fig. 1

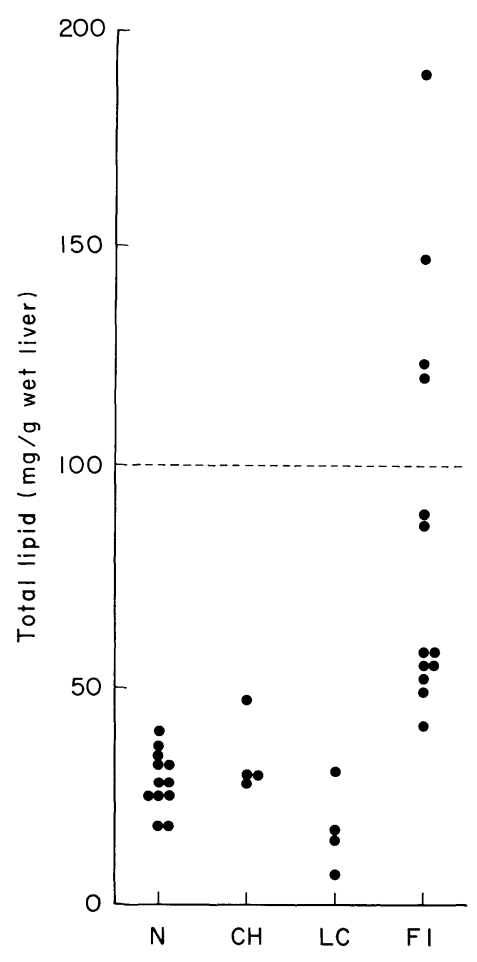

Fig. 2

Fig. 1. CTN in various diffuse liver diseases.

Abbreviations: N, normal control; CH, chronic hepatitis; LC, liver cirrhosis; FI, fatty infiltration.

Fig. 2. Total lipid content in various diffuse liver diseases. Horizontal broken line denotes biochemical criterion for fatty liver, i.e., total lipid content above $100 \mathrm{mg} / \mathrm{g}$ wet liver. Abbreviations are the same as Fig. 1 .

Correlation between CTN and total lipid content of the liver

A significant correlation existed between CTN and total lipid content $(r=-0.89$; $p<0.001$ ). In Fig. 3, vertical and horizontal broken lines represent normal control levels of CTN and total lipid content, respectively. Two cases around the crossing of both lines are those with no fatty infiltration. If the diagnostic criterion for the fatty liver was set at total lipid content above $100 \mathrm{mg} / \mathrm{g}$ wet liver, CT criterion was estimated at CTN below $48 \mathrm{H}$ from the regression formula (Fig. 3). 


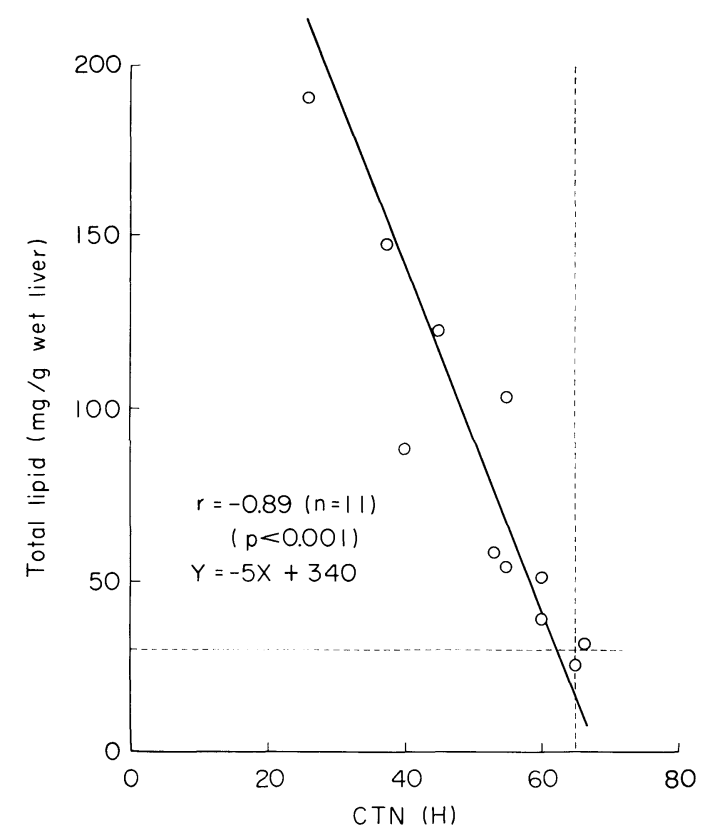

Fig. 3. A relation of CTN to total lipid content. Vertical and horizontal broken lines represent normal control levels of CTN and total lipid content, respectively.

Fig. 4. Comparison of semiquantitative grading of fatty infiltration to total lipid content and CTN. Abbreviations; L, fatty infiltration below $30 \%$ of the hepatic lobule; M, from $30 \%$ to $50 \%$; $\mathrm{H}$, above $50 \%$.

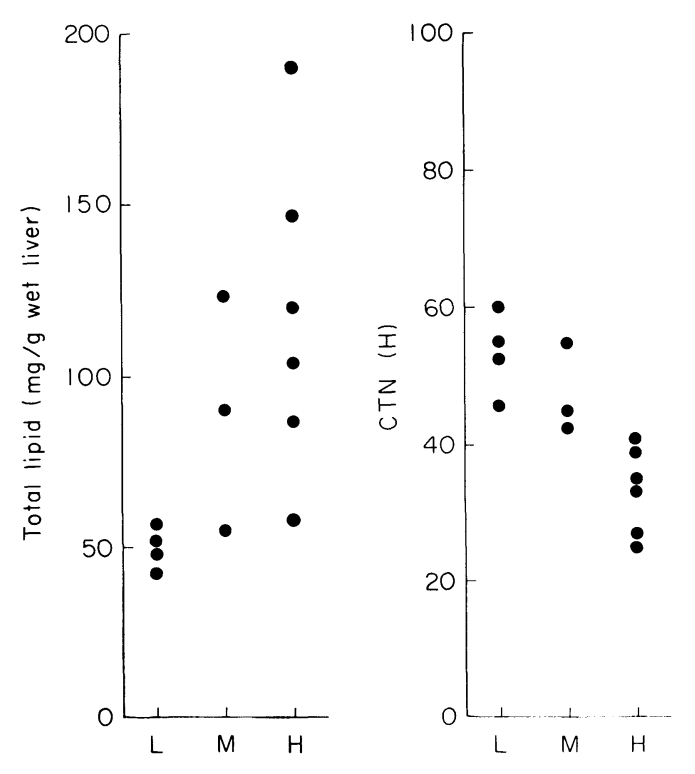

Comparison of semiquantitative grading of fatty infiltration to total lipid content and CTN

Semiquantitative classification of fatty infiltration was as follows: fatty infiltration below $30 \%$ of the hepatic lobule was graded low $(\mathrm{L}), 30-50 \%$ graded moderate $(\mathrm{M})$, above $50 \%$ graded high $(\mathrm{H})$. Fatty infiltration had a positive correlation to the total lipid content and a negative correlation to CTN. But considerable 
overlapping was seen among three groups. If the histological criterion of fatty liver was defined as fatty infiltration above $50 \%$ of the lobule, $\mathrm{H}$ group was equivalent to fatty liver. If the biochemical criterion of fatty liver was defined as total lipid content above $10 \%$ of the wet liver weight, the rate of conformity between histological and biochemical criteria was four to six, i.e. 67\% (Fig. 4).

\section{Discussion}

There are many reports which insist on the usefulness of computed tomography in the diagnosis of fatty liver. Tada et al. (1979) investigated CTNs of various diffuse liver diseases and found that all types of diffuse liver diseases had lower CTNs than normal control and a negative correlation existed between grading of fatty infiltration in three groups and their CTNs. Yamawaki et al. (1981) measured fatty infiltration with morphometric approach and showed a significant correlation between CTN and the degree of fatty infiltration $(t=-0.746 ; p<0.01)$. Ducommun et al. (1979) produced fatty liver in rabbits by carbon tetrachloride $\left(\mathrm{CCl}_{4}\right)$ and found that a significant correlation existed between decrease in CTN and increase in triglyceride concentration $(r=-0.78 ; p<0.001)$. With regard to the spread in CTN difference of $\pm 12 \mathrm{H}$ (the standard error of the estimate of the linear approximation), they discussed that because the rabbit's liver was so small and flat, its boundaries were sometimes difficult to detect, therefore, resultant CT averaging of nonhepatic and hepatic tissues would contributed to experimental error.

The best way to study the correlation of fatty infiltration and CTN seems to be a biochemical measurement of total lipid content of the liver in men. In this regard it should not be forgotten that fatty deposits would be removed quickly from the liver with adequate therapy. Therefore in the study of the correlation between CTN and fatty infiltration, CT scanning and liver biopsy should be performed as closely as possible.

We tried to make a strict approach in adopting biochemical measurement of total lipid content in just human livers and lessening the interval between the two examinations. As a result, we had a better correlation than those of previous reports $(r=-0.89 ; p<0.001)$.

Previously reported CT criteria of fatty liver were a reversal of the white (liver)-to-black (vessels) appearance (Schere et al. 1979), and a reversal of the normally higher density of the liver than the spleen (Tada et al. 1979). These criteria indicated analogical approach toward the study, and now we translate them into digital approach using our data. As mean CTN of the aorta which represented CTN of the blood was about $40 \mathrm{H}$, the former criterion was translated as CTN below $40 \mathrm{H}$, and as the mean CTN of the spleen was about $50 \mathrm{H}$, the latter criterion was translated as CTN below $50 \mathrm{H}$. By the way, Fig. 1 shows that CTN below $50 \mathrm{H}$ cannot be explained without fatty infiltration and never means fatty liver itself. If the diagnostic criterion of the fatty liver was set at total lipid content about $100 \mathrm{mg} / \mathrm{g}$ wet liver, CT criterion was estimated at CTN below $48 \mathrm{H}$ from the 
regression formula with $\pm 5.7 \mathrm{H}$ standard error of the estimate.

These results have the same implication as a reversal of CTN of the liver to the spleen previously mentioned.

Schere et al. (1979) reported that only a combination of both findings, decreased CTN and clearly visible, more radiodense intrahepatic vessels, was pathognomonic for severe fatty infiltration of liver cell, and that the combination of both findings was present only in specimens that showed a percentage of 70 or more of fatty infiltration of liver cells. Based on these criteria, many cases of fatty liver would be missed because histological criterion of fatty liver is usually taken as fatty infiltration above $50 \%$ in the hepatic lobule. Therefore, digital approach using CTN will be necessary and this study will bring a firm basis for it.

\section{Acknowledgment}

We thank Assistant Professor Y. Maruhama, the First Department of Internal Medicine, Iwate Medical University, for kindly helping us in the measurement of total lipid content of the liver.

\section{References}

1) Ducommun, J-C., Goldberg, H.I., Korobkin, M., Moss, A.A. \& Kressel, H.Y. (1979) The relation of liver fat to computed tomography numbers: a preliminary experimental study in rabbits. Radiology, 130, 511-513.

2) Folch, J., Ascoli, I., Less, M., Meath, J.A. \& Lebaron, F.N. (1951) Preparation of lipide extracts from brain tissue. J. biol. Chem., 191, 833-841.

3) Frings, C.S., Flendley, T.W., Dunn, R.T. \& Qeen, C.A. (1972) Improved determination of total serum lipids by the sulfo-phospho-vanillin reaction. Clin. Chem., 18, 673-674.

4) Kalk, H. (1965) Über die Fettleber. Munch. med. Wochenschr., 107, 1141-1147.

5) Kobayakawa, K. (1959) On the studies of lipid fraction in the fatty liver and fatty cirrhosis. Tr. Soc. Path. Jap., 48, 1518-1544. (in Japanese with English abstract)

6) Okudaira, M. (1980) Fatty liver - From the standpoint of pathology. Acta hepatol. jap., 21, 914. (Japanese)

7) Schere, U., Santos, M. \& Lissner, J. (1979) CT studies of the liver in vitro: a report on 82 cases with pathological correlation. J. comput. assist. Tomogr., 3, 589-595.

8) Tada, A., Shikae, M., Morinaga, K., Morikawa, T., Nei, H., Aburano, T. \& Hisada, K. (1979) Evaluation of diffuse hepatic disease by computed tomography. Part 1. Diagnosis and quantitative analysis of fatty liver by CT values. Clin. Radiology, 24, 1319-1324. (in Japanese with English abstract)

9) Yamawaki, T., Hirofuji, H., Yatomi, A., Kawabe, M. \& Sugie, H. (1981) The study of fatty infiltration of the liver with the use of CT scan. Acta hepatol. jap., 22, 236-243. (in Japanese with English abstract) 